\section{Inhalative Steroide verhindern Klinikeinweisungen}

\section{Asthmatiker, die anläßlich einer Hospitalisierung eine Therapie mit inhalativen Kortikosteroiden beginnen, haben eine dreifach bessere Chance, in den folgenden 3 Monaten nicht noch ein weiteres Mal in der Klinik aufgenommen zu werden.}

Hintergrund der Studie von Lucie Blais und Mitarbeitern vom Royal Victoria Hospital in Montreal ist die Tatsache, daß die zunehmende Verwendung von inhalativen Steroiden zur präventiven Behandlung des Asthma bronchiale bisher nicht zu einer Umkehr der Tendenz zu immer schwereren Verläufen der Erkrankung geführt hat. Weltweit erkranken immer mehr Menschen immer heftiger an Asthma.

\section{Kortikoide wirken protektiv - aber erst nach einer Latenzzeit}

Die kanadischen Epidemiologen werteten nun Daten der staatlichen Gesundheitskasse von Saskatchewan aus, über die $95 \%$ der Bevölkerung krankenversichert sind. Zwischen 1977 und 1993 waren 2059 der Versicherten wenigstens einmal wegen einer Asthmaerkrankung in der Klinik behandelt worden. Davon wurden 482 innerhalb eines Jahres ein zweites Mal aufgenommen.

Die Frage lautete: Sind diese Asthmatiker seltener mit inhalativen Steroiden behandelt worden? Dies wäre ein Hinweis auf eine protektive Wirkung der Steroide.

Tatsächlich konnte ein derartiger Zusammenhang für Patienten ermittelt werden, wenn zwischen den beiden Klinikaufenthalten 16 Tage bis 3 Monate lagen. Hier senkte die Therapie mit Steroiden das Risiko hinsichtlich einer erneuten Klinikeinweisung um ein Drittel. Wenn die zweite Einweisung innerhalb von 15 Tagen nach der ersten erfolgte, war jedoch kein Unter- schied zwischen den Steroidverordnungen nachweisbar. Blais und Mitarbeiter schließen daraus, daß es mindestens 2 Wochen dauert, bis die Steroide ihre protektive Wirkung entfalten.
Die protektive Wirkung war auch nach dem dritten Monat noch nachweisbar. Der Zusammenhang wurde jedoch immer schwächer: Nach einem halben Jahr war er nicht mehr signifikant. Dies könnte bedeuten, daß Sterove Wirkung haben.

Die Autoren vermuten allerdings, vorliegen könnte. Es sei wahrscheinlich, daß Patienten mit einem günstigen Verlauf der Erkrankung die inhalativen Steroide nach einiger Zeit (entgegen dem Rat ihrer Ärzte) absetzen. Damit steigt der Anteil der schweren Fälle unter den Steroidbenutzern - und für diese Patientenklientel ist das Risiko einer Re-Hospitalisierung natürlich erhöht.

(rme)

Blais $\mathrm{L}$ et al.: Inhaled corticosteroids and the prevention of Beadmission to hospital for asthma. Am J Respir Crit Care Med 1998; 158: 126-32. ide nur eine zeitlich begrenzte protektidaß hier eine systematischer Fehler

\section{Cave: Kortikosteroide bei Diabetikern}

Vorsicht ist geboten, will man einem Diabetiker Kortikosteroide verordnen. Denn die Diabetes-Einstellung kann von in hohen Dosen inhaliertem Fluticason gestört werden, wie der Fall eines 67 Jahre alten, seit 10 Jahren an Asthma leidenden Mannes zeigt. Viermal täglich inhalierte er $0,5 \mathrm{mg}$ Ipratropiumbromid und $5 \mathrm{mg}$ Salbutamol. Da er seit 40 Jahren Typ-2-Diabetes hatte, nahm er täglich 5 mg Glibenclamid und 1700 mg Metformin. Ansonsten erhielt er keine weiteren Medikamente ein, rauchte nicht, trank nicht und hielt sich strikt an die ärztlichen Verordnungen.

Nun begann er eine Asthmatherapie, in der er täglich $2000 \mu$ g Fluticasonpropionat inhalierte. Nach Beginn der Fluticason-Einnahme verbesserten sich zwar die subjektiven Atembeschwerden, und die durchschnittliche wöchentliche maximale Flußgeschwindigkeit stieg von 410 1/min auf 440 $1 /$ min.

In der 3. Woche entwickelte der Patient jedoch eine persistierende Glukosurie. Da alle gemessenen Glukose-Parameter im Urin in Woche 3 und 4 positiv waren, wurde die Fluticason-Dosis langsam (bis Woche 14) auf $500 \mu \mathrm{g}$ pro Tag reduziert. Daraufhin besserte sich die Diabetes-Einstellung ab Woche 15 schlagartig. Anschließend wurde die Fluticason-Dosis in Woche 25 auf $1000 \mu \mathrm{g}$ erhöht. Innerhalb einer Woche entwickelte der Patient erneut eine Glukosurie, die nach Dosisreduzierung auf $500 \mu \mathrm{g}$ wiederum verschwand.

Dieser Fall dokumentiert, daß die Inhalation von hochdosiertem Fluticason zu Glukosurie und zu einem dosisabhängigen Anstieg der Glykohämoglobin-Konzentration führen kann. Der $\mathrm{HbA}_{1 c}$-Wert betrug 8,2\% nach 2000 $\mu \mathrm{g}$ bzw. 7,8\% nach $1000 \mu \mathrm{g}$ Fluticason täglich. Wenn Diabetiker Fluticasonpropionat in hohen Dosen zur Inhalation erhalten, sollte daher immer daran gedacht werden, daß diese Kortikosteroide die Diabetes-Einstellung beeinflussen können. 